\title{
Catalog of Radio Galaxies with $z>0.3$. III: Angular Sizes and Flux Density According to the NVSS Data
}

\author{
M.L. Khabibullina ${ }^{a}$, O.V. Verkhodanov ${ }^{a}$ \\ Special Astrophysical Observatory of the Russian AS, Nizhnij Arkhyz 369167, Russia
}

Received December 15, 2008; accepted April 4, 2009.

\begin{abstract}
We describe the procedure of the construction of a sample of distant $(z>0.3)$ radio galaxies using the NED, CATS and SDSS databases for further use in various statistical tests. We believe the sample to be free of objects with quasar properties. This paper is the third part of the description of the radio galaxies catalog that we plan to use for cosmological tests. We report the results of the sample of angular sizes for the NVSS survey list objects, and its preliminary statistical analysis. Three-parameter diagrams "angular size-redshift-flux density" and "angular size-redshift-spectral index", and their two-parameter projections are constructed. Three subsamples of radio galaxies are separated in the "source size-flux density" diagram.
\end{abstract}

PACS: 98.54.Gr, 98.62.Py, 98.70.Dk, 98.80.Es

\section{INTRODUCTION}

The present work is the third part of the catalog of radio galaxies located within $0.3<z<5.2$ redshifts. The first two parts [1, 2] (further, Papers I and II) describe the way the sample was constructed, based on the NED 1 , CATS2, and $\operatorname{SDS} \sqrt{3}$ data, including an analysis of other works with the aim of excluding the objects with quasar properties. Paper I lists 2442 objects with their equatorial coordinates for epoch J2000.0, spectroscopic redshifts, and spectral indices, deduced according to the cross-identification results of the selected radio galaxies with the CATS radio catalogs, and computed at 325, 1400 and $4850 \mathrm{MHz}$. The list of objects in Paper I also contains the names of the initial catalogs from where we adopted the data for the objects (and the names of radio sources in case of the $3 \mathrm{C}$ and $4 \mathrm{C}$ surveys). Paper II presents photometric data from the NED and SDSS archives. We constructed the "magnitude-redshift" Hubble diagrams for 11 filters. For the SDSS survey [3] (g, i, $\mathrm{r}$, z passbands) we determined the parameters of linear regression on the Hubble diagram. The preparation of the catalog presented in the three papers is the first step for making cosmological tests on the observed physical parameters of radio galaxies at different epochs.

An objective of our catalog was a manifold in-

\footnotetext{
1 http://nedwww.ipac.caltech.edu

2 http://cats.sao.ru

3 http://sdss.org
}

crease of the comparatively uniform sample of radio galaxies, which would allow increasing the accuracy of the determination of cosmological parameters in an independent set of tests, apart from the cosmic microwave background and a large-scale structure. In the last part of the catalog, which is supplemented with this paper, we give the list of objects with their angular sizes from the NVSS survey 4 and the flux density measurements data at $1400 \mathrm{MHz}$.

The radio source characteristics collected in this list can be used in the "radio luminosity-redshift" and "angular size-redshift" tests. Both tests are connected with the galaxy characteristics that greatly evolve. Hence, the evaluation of the cosmological parameters, based on them, has a large dispersion. Nevertheless, the "angular size-redshift" (or " $\theta-z$ ") diagram is widely used in the papers, that consider both the minimal size of point sources [5], and the average angular size of a radio galaxy at a given redshift, corrected for evolutionary effects $[6$. The angular size of a radio source depending on its redshift can be used in a combined test with the spot sizes in the cosmic microwave background 7, where both the scale of the CMB fluctuations, and the characteristic sizes of millisecond sources are used.

Note once more that all the methods of parameter finding from the relation " $\theta-z$ " are aggravated with uncertainties linked with evolutionary effects, and the extended objects, in addition, with the task of de-

4 http://www.cv.nrao.edu/nvss/ 


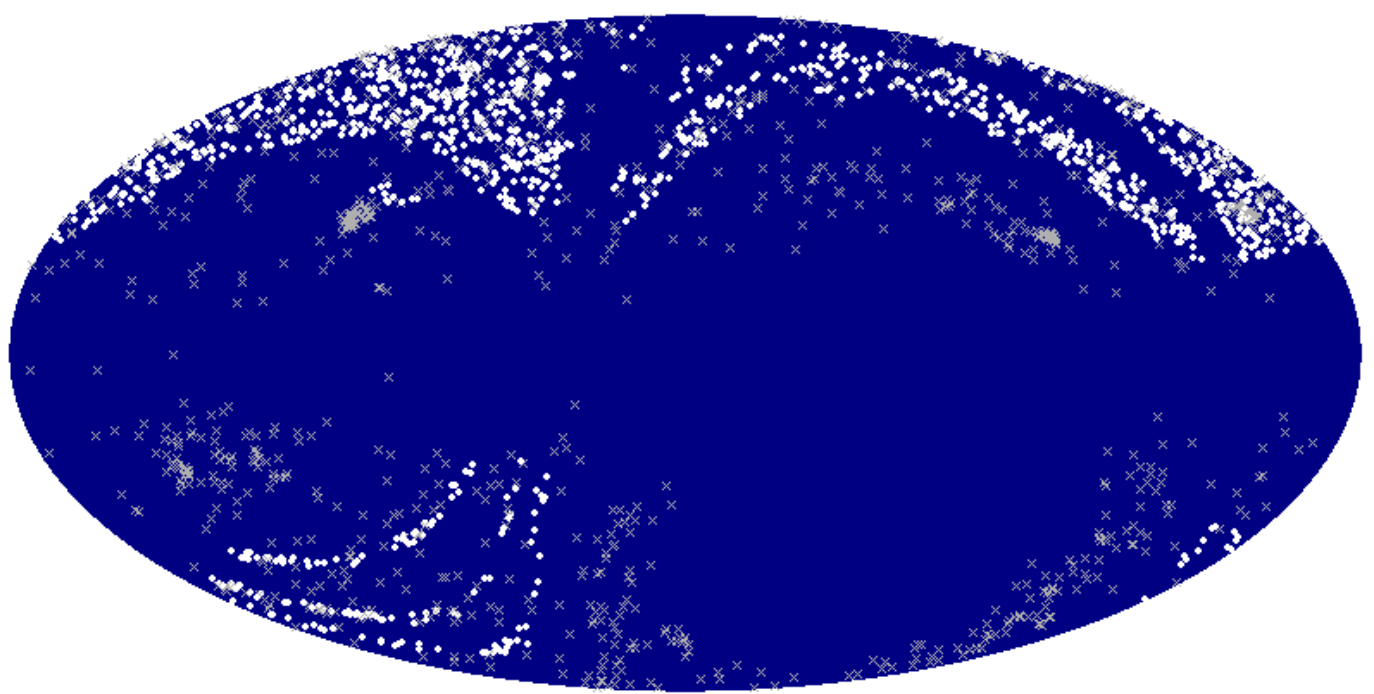

Figure 1: Sky positions of selected radio sources in Galactic coordinates. Objects location is determined by the simultaneous presence of observational data in optical and radio ranges. White circles indicate SDSS objects and gray crosses mark all other sources.

termining the outer edge of an object. Nevertheless, using the source size as a standard ruler is justified. If radio galaxies have a fundamental plane, determined by such physical parameters as, e.g., the mass of the radio galaxy or its central black hole, which was already mentioned in Paper II, then we have reason to link the angular size of the source with the history of formation of the active nucleus, described by a set of standard measurable parameters, like the accretion velocity, angular momentum, magnetic field magnitude, and mass of the central black hole. This gives us grounds to expect that the proposed in the literature approaches of parameter estimation of the cosmological model will work as well. Note that the highest certainty of parameter estimation may be reached via a combination of independent tests (for radio galaxies, see, e.g., [8, 9, 10]).

Describing the catalog in Papers I and II, we made a preliminary statistical analysis, and constructed the Hubble diagrams, describing the behavior of different parameters with the change in redshift. In the present work, devoted to the construction of a sample, and a statistical analysis of the radio galaxy catalog, we collected the objects with known spectroscopic redshifts and with measured angular sizes. To make the sample uniform, we adopted the angular sizes from the NVSS catalog 4, obtained on the Very Large Array (VLA) with the beam of $45^{\prime \prime}$. In further studies we shall as well use the angular size data from other surveys, like, for example, the FIRST survey [11] data with the beam of $5^{\prime \prime}$. Below we describe our sample, make its statistical analysis and construct diagrams for var- ious physical parameters depending on redshifts.

\section{THE CATALOG}

\subsection{Description of the Catalog}

The data are presented in the Table, where the columns give the object names, the equatorial coordinates of the galaxies from the NVSS survey for epoch J2000.0, linear sizes (or upper limits) on two axes in arc seconds, flux densities in mJy according to the NVSS data, and redshifts. Sky positions of selected radio sources are shown in Fig. 1. The complete catalog is available from the CATS databas 5 , and CDS ftp6, CDS database7.

\subsection{Statistical Analysis of the Sample}

We already pointed out in previous papers that our list of galaxies is not complete and homogeneous, as the sample contains objects drawn from different catalogs and unconnected sky areas. The most complete and homogeneous subsample of the list contains objects from the SDSS survey, marked in Fig. 1 by white circles. The SDSS radio galaxies include many objects with small redshifts $(z<0.5)$ and small flux densities $(S<15 \mathrm{mJy})$, making this subsample distinguish itself quality-wise among other subsamples.

\footnotetext{
5 ftp://cats.sao.ru/pub/CATS/RGLIST

6 ftp://cdsarc.u-strasbg.fr/pub/cats/J/other/AstBu/64.357

7 http://cdsarc.u-strasbg.fr/cgi-bin/VizieR?-source=J/other/AstBu/64.357
} 


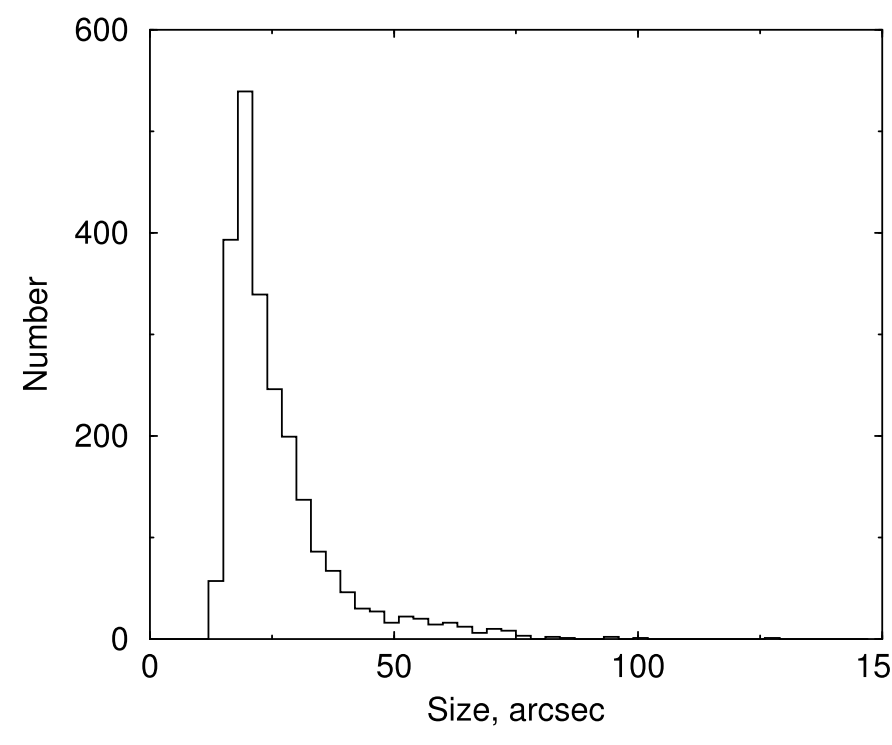

Figure 2: Histogram of the source angular sizes distribution.

Using the resulting catalog, we made a preliminary statistical analysis of the chosen objects. Figs. 2-4 demonstrate the results of this analysis.

Figure 2 represents the histogram of the sources major axes distribution by angular size. The histogram peak's position de facto corresponds to the size of point sources in the NVSS survey after their reduction with the beam of $45^{\prime \prime}$.

$\begin{array}{lccr}\text { We } & \begin{array}{c}\text { constructed } \\ \text { size-redshift-flux }\end{array} & \text { the } & \begin{array}{c}\text { "source } \\ \text { density" }\end{array} \\ \text { and } & \text { "angular } & & \text { size-redshift-spect- }\end{array}$

ral index" (Fig.4) diagrams, and some projections onto the parametric planes thereof. Spectral index was computed for the frequency of $1400 \mathrm{MHz}$ in Paper I.

For the ratio "source size-redshift-flux density", we made two projections in the planes "source sizeflux density", and "source size-redshift". For the first projection " $\theta-S$ ", we isolated three areas with different radio source densities, corresponding to the unresolved sources from the NVSS, extended radio objects, and gigantic radio galaxies. The area boundaries can be provisionally described by the curves (Fig.3, solid lines): (1) separating the unresolved and extended radio galaxies as $\theta=28.3 \times \exp (-3 p)+19.3$, where $p=\ln (S / 8.4), \theta$ is the angular size in arc seconds, $S$ is the flux density in mJy; (2) separating the extended and gigantic radio galaxies as $\theta=97.4-5.5 \ln (S)$. Different symbols mark different redshift ranges: circles represent the $0.3<z<0.7$ range, squares mark the $0.7 \leq z<1.5$ range, pluses mark $1.5 \leq z$. Note that the bulk of far galaxies from our sample (marked with bright gray) is located at the flux density range from 40 to 300 mJy at $1400 \mathrm{MHz}$.

The bottom source separation on the "source sizeflux density" diagram is of course preconditioned by the selection effect, defined by the size of the VLA directivity pattern in the NVSS survey. For the study of sources from this area some better resolution data are needed, for example, from the FIRST [11 survey, as well conducted on the VLA, but with the beam of 5", or from the observations of different sources on VLBA or VLBI. Besides, the NVSS data may contain some particularities, linked with the source size determination at the noncircular directivity pattern [12. Nevertheless, the parameters of the objects (extended radio sources) from the top and middle parts of the diagram are applicable for further use in cosmological tests.

On the second projection "source size-redshift" (" $\theta-z ")$, we separated two object populations, marked with different symbols: triangles mark the SDSS sources, and circles indicate the rest of the objects. One can see from the figure that the SDSS radio galaxies mainly belong to the small $z$ range in our sample, having a flux density scatter from the minimal to the maximal values. Note that the bigger radio galaxies are of interest in estimating the extended objects contribution into the microwave background [13].

For the "source size-redshift-spectral index" relation (Fig. 4) we made only one projection - "spectral index-source size", presented in two variants. In the first one the triangles mark the SDSS objects, in the second one different symbols mark objects with different redshift ranges: circles- $0.3<z<0.7$, squares- $0.7 \leq z<1.5$, pluses mark the objects with $1.5 \leq z$. The diagram demonstrates a relationship between the redshift of the source and the spectral index in radio range, discovered long ago [14, 15], what was already discussed in Paper I. For another thing, we observe the expected relation-bigger sources have steeper spectra due to the synchrotron emission in radio lobes. Note that for the radio galaxies discovered in the SDSS, the value of the spectral index is smaller, while the distribution on the source size does not bear any great differences. This difference is explained by the selection effect and an existence of the "spectral index-redshift" relation. This latter fact is demonstrated on the right bottom figure from Fig. 4, where the region of large $z$ is marked in light gray. 


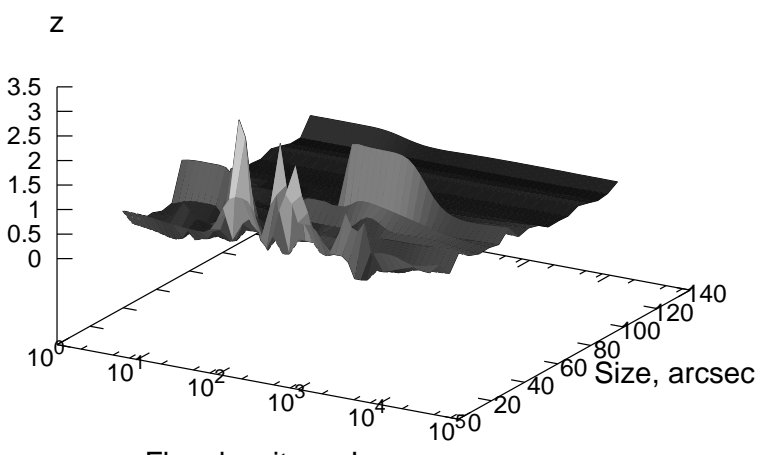

Flux density, mJy
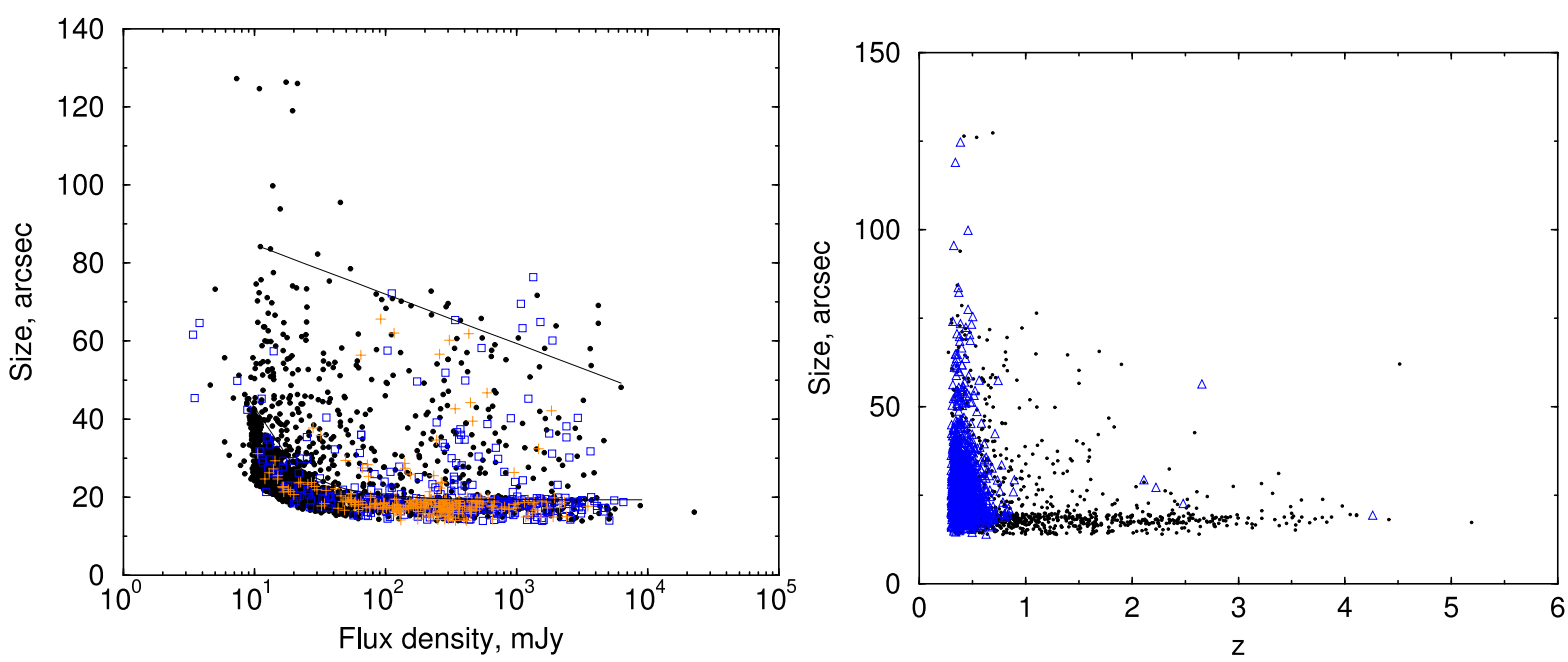

Figure 3: Top: "angular size-redshift-flux density" relation for radio galaxies. Bottom left: "source size-flux density" relation. Different symbols mark different redshift ranges: circles-0.3<z<0.7, squares-0.7 $\leq$ $z<1.5$, pluses $-1.5 \leq z$. Solid lines divide the areas of different density. Bottom right: "source size-redshift" relation. Triangles indicate the SDSS data. Circles mark the objects from all other catalogs.

\section{CONCLUSIONS}

We report the final part of the radio galaxies cata$\log$ with redshifts $z>0.3$, which contains complete information on the angular sizes of the objects, and flux densities at $1400 \mathrm{MHz}$. The catalog is based on lists of objects from the NED and CATS databases. Our complete sample includes the SDSS objects, and the objects from the "Big Trio" program [16, 17. We perform a preliminary statistical analysis of the angular size data, flux density and spectral index calculations. On the diagram "source size-flux density" we separated three radio galaxy subsamples, varying in angular sizes and flux densities. We approximate the boundaries of these subsamples based on analytic functions. The bottom object separation on the "source size-flux density" diagram is preconditioned by the selection effect defined by the size of the VLA directivity pattern in the NVSS survey. In order to use the sources from this area in the cosmological tests, connected with the source size, some better resolution data are needed.

\section{Acknowledgments.}

This research has made use of the NASA/IPAC Extragalactic Database (NED) which is operated by the Jet Propulsion Laboratory, California Institute of Technology, under contract with the National Aeronautics and Space Administration. We also made use of the CATS database of radio astronomical catalogs [18, 19] and FADPS8 radio- $^{8}$ astronomical data reduction system [20, 21]. The authors are grateful to Olga Kashibadze (the SAO RAS) for the aid in nonlinear function approximation using the MATLAB package. This work was supported by the Program of

8 http://sed.sao.ru/ vo/fadps_e.html 

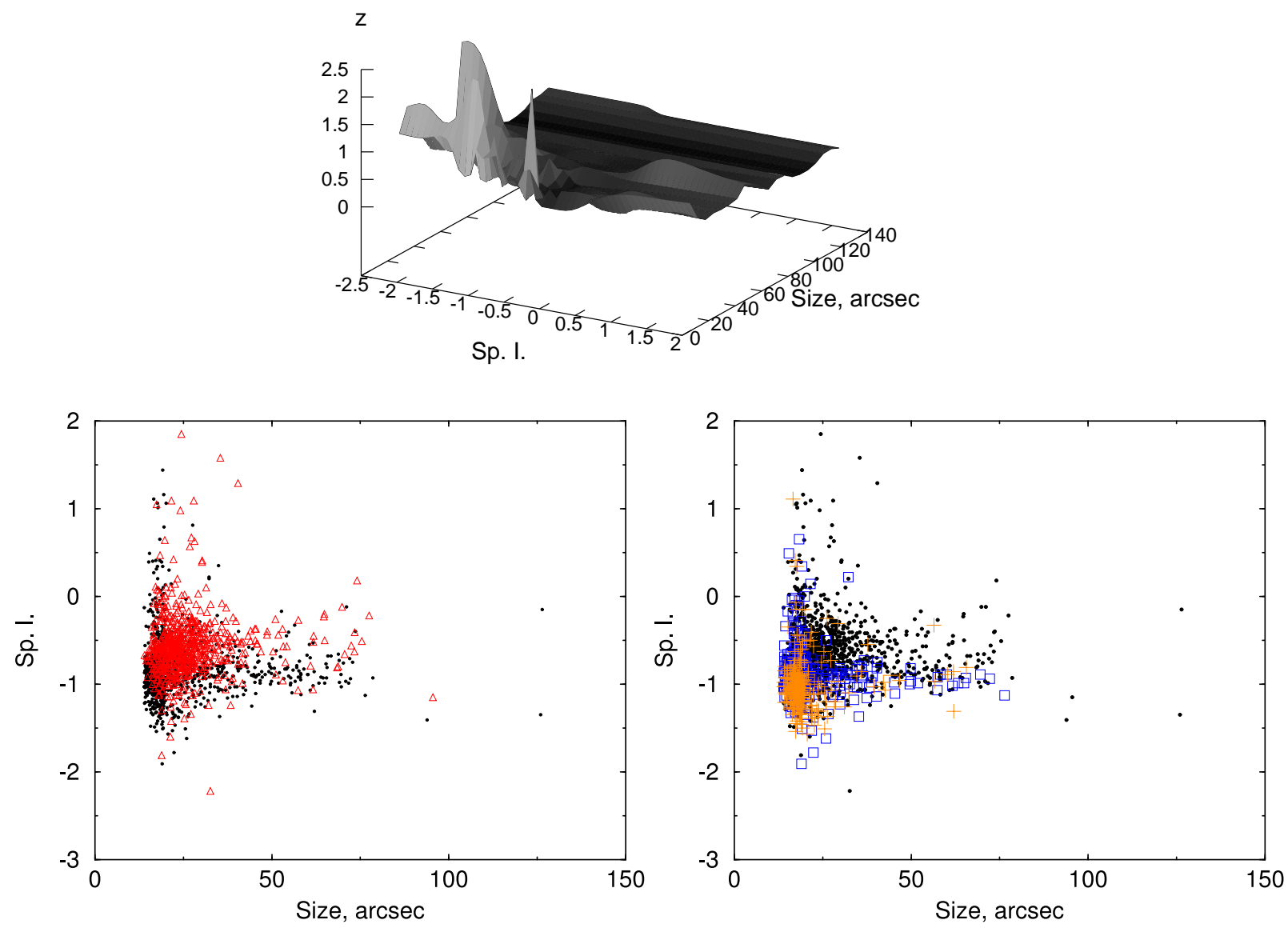

Figure 4: Top: "source size-redshift-spectral index" relation for radio galaxies. The spectral index is computed at $1400 \mathrm{MHz}$. Bottom left: "spectral index-source size" relation. Triangles mark the SDSS data, circles represent the objects from all other catalogs. Bottom right: again, "spectral index-source size" relation. Different symbols mark different redshift ranges: circles $-0.3<z<0.7$, squares $-0.7 \leq z<1.5$, pluses $-1.5 \leq z$.

the Support of Leading Scientific Schools in Russia (the School of S. M. Khaikin) and the grants of the Russian Foundation of Basic Research (project nos. 09-02-00298 and 09-02-92659-IND). O.V.V. acknowledges partial support from the RFBR (project No 07-02-01417-a), and the Fund for Support of National Science ("Young PhDs of the RAS" program).

\section{References}

[1] M. L. Khabibullina and O. V. Verkhodanov, Astrophys. Bull. 64, 123 (2009), astro-ph/0911.3741.

[2] M. L. Khabibullina and O. V. Verkhodanov, Astrophys. Bull. 64, 276 (2009), astro-ph/0911.3747.

[3] D. P. Schneider, P. B. Hall, G. T. Richards et al., AJ 134, 102 (2007).

[4] J. J. Condon, W. D. Cotton, E. W. Greisen et al., AJ 115, 1693 (1998).
[5] L. I. Gurvits, K. I. Kellermann and S. Frey, A\&A 342, 378 (1999).

[6] E. J. Guerra, R. A. Daly and L. Wan, ApJ 544, 659 (2000).

[7] J. C. Jackson and A. L. Jannetta, JCAP 11, 002 (2006).

[8] O. V. Verkhodanov and Yu. N. Parijskij, Bull. SAO 55, 66 (2003).

[9] O. V. Verkhodanov and Yu. N. Parijskij, in Proc. 14th Internat. School, "Particles and Cosmology", Ed. by S. V. Demidov, V. A. Matveev, and V. A. Rubakov (Inst. Nucl. Phys. RAS, Moscow, 2008) p. 109.

[10] O. V. Verkhodanov and Yu. N. Parijskij, Radio galaxies and Cosmology, (Fiz.Mat.Lit., Moscow, 2009) [in Russian].

[11] R. L. White, R. H. Becker, D. J. Helfand and M. D. Gregg, ApJ 475, 479 (1997).

[12] A. E. Kimball and Z. Ivezic, AJ 136, 684 (2008).

[13] O. V. Verkhodanov, M. L. Khabibullina, M. Singh 
et al., in "Practical Cosmology", Proc. Internat. Conf. "Problems of Practical Cosmology", Ed. by Yu. Baryshev, I. N. Taganov, P. Teerikorpi, Russian Geograph. Soc., St.Petersburg, p. 247 (2008).

[14] R. D. Dagkesamanskii, Nature, 226, 432 (1970).

[15] G. Blumenthal and G. Miley, A\&A 80, 13 (1979).

[16] Yu. N. Parijskij, W. M. Goss, A. I. Kopylov et al., Bull. SAO 40, 5 (1996).

[17] Yu. N. Parijskij, W. M. Goss, A. I. Kopylov et al., Astron. Astrophys. Trans. 18, 437 (1999).

[18] O. V. Verkhodanov, S. A. Trushkin, H. Andernach, and V. N. Chernenkov, in "Astronomical Data Analysis Software and Systems VI", Ed. by G.Hunt and H.E.Payne, ASP Conf. Ser., 322, 46 (1997).

[19] O. V. Verkhodanov, S. A. Trushkin, H. Andernach and V. N. Chernenkov, Bull. SAO 58, 118 (2005), arXiv:0705.2959

[20] O. V. Verkhodanov, in "Astronomical Data Analysis Software and Systems VI", Ed. by G.Hunt and H.E.Payne, ASP Conf. Ser., 125, 46 (1997).

[21] O. V. Verkhodanov, B. L. Erukhimov, M. L. Monosov et al., Bull. SAO 36, 132 (1993). 